IOS Press

\title{
Prognostic significance of Wilms tumor 1 mRNA expression levels in peripheral blood and bone marrow in patients with myelodysplastic syndromes
}

\author{
Sumiko Kobayashia ${ }^{\mathrm{a}, *}$, Yasunori Ueda ${ }^{\mathrm{b}}$, Yasuhito Nannya ${ }^{\mathrm{c}}$, Hirohiko Shibayama ${ }^{\mathrm{d}}$, Hideto Tamura $^{\mathrm{e}}$, \\ Kiyoyuki Ogata ${ }^{\mathrm{e}, \mathrm{f}}$, Yoshiki Akatsuka ${ }^{\mathrm{g}}$, Kensuke Usuki ${ }^{\mathrm{h}}$, Yoshikazu Ito ${ }^{\mathrm{i}}$, Masaya Okada $^{\mathrm{j}}$, \\ Takahiro Suzuki $^{\mathrm{l}}$, Tomoko Hata ${ }^{\mathrm{l}}$, Akira Matsuda ${ }^{\mathrm{m}}$, Kaoru Tohyama ${ }^{\mathrm{n}}$, Keiji Kakumoto ${ }^{\circ}$, Daisuke Koga ${ }^{\mathrm{p}}$, \\ Kinuko Mitani ${ }^{\mathrm{q}}$, Tomoki Naoe ${ }^{\mathrm{r}, \mathrm{s}}$, Haruo Sugiyama ${ }^{\mathrm{t}}$ and Fumimaro Takaku ${ }^{\mathrm{u}}$ \\ ${ }^{\mathrm{a}}$ Department of Hematology and Rheumatology, Nihon University School of Medicine, Tokyo, Japan \\ ${ }^{\mathrm{b}}$ Department of Haematology/Oncology, Transfusion and Haemapheresis Center, Kurashiki Central Hospital, \\ Okayama, Japan \\ ${ }^{\mathrm{c}}$ Department of Hematology and Oncology, Graduate School of Medicine, The University of Tokyo, Tokyo, Japan \\ ${ }^{\mathrm{d}}$ Department of Hematology and Oncology, Osaka University Graduate School of Medicine, Osaka, Japan \\ e Division of Hematology, Department of Medicine, Nippon Medical School, Tokyo, Japan \\ ${ }^{\mathrm{f}}$ Department of Hematology, Shin-Yurigaoka General Hospital, Kanagawa, Japan \\ ${ }^{\mathrm{g}}$ Department of Hematology, Fujita Health University School of Medicine, Aichi, Japan \\ ${ }^{\mathrm{h}}$ Department of Hematology, NTT Medical Center Tokyo, Tokyo, Japan \\ ${ }^{\mathrm{i}}$ Department of Hematology, Tokyo Medical University Hospital, Tokyo, Japan \\ ${ }^{\mathrm{j}}$ Division of Hematology, Department of Internal Medicine, Hyogo College of Medicine, Hyogo, Japan \\ ${ }^{\mathrm{k}}$ Division of Hematology, Department of Medicine, Jichi Medical University, Tochigi, Japan \\ ${ }^{1}$ Department of Hematology, Atomic Bomb Disease Institute, Nagasaki University Graduate School of Biomedical \\ Sciences, Nagasaki, Japan \\ ${ }^{\mathrm{m}}$ Department of Hemato-Oncology, Saitama International Medical Center, Saitama Medical University, Saitama, \\ Japan \\ ${ }^{\mathrm{n}}$ Department of Laboratory Medicine, Kawasaki Medical School, Okayama, Japan \\ ${ }^{\circ}$ Information Management Office, Drug Safety Research Center, Tokushima Research Institute, Otsuka \\ Pharmaceutical Co., Ltd., Tokushima, Japan \\ ${ }^{\mathrm{p}}$ Diagnostic Division, Otsuka Pharmaceutical Co., Ltd., Tokushima, Japan \\ ${ }^{\mathrm{q}}$ Department of Hematology and Oncology, Dokkyo Medical University School of Medicine, Tochigi, Japan \\ ${ }^{\mathrm{r}}$ Department of Hematology and Oncology, Nagoya University Graduate School of Medicine, Nagoya, Japan \\ ${ }^{s}$ National Hospital Organization Nagoya Medical Center, Nagoya, Japan \\ ${ }^{\mathrm{t}}$ Department of Functional Diagnostic Science, Osaka University Graduate School of Medicine, Osaka, Japan \\ u Japanese Association of Medical Sciences, Tokyo, Japan
}

\footnotetext{
${ }^{*}$ Corresponding author: Sumiko Kobayashi, Department of Hematology, Hematopoietic Cell Transplantation, 35-2, Sakae-cho, Itabashi-ku, Tokyo 173-8610, Japan. Tel.: +81 3964 1161; Fax: +81 3964 1164; E-mail: sumiko_kobayashi@tmghig.jp.
}

ISSN 1574-0153/16/\$35.00 (c) 2016 - IOS Press and the authors. All rights reserved This article is published online with Open Access and distributed under the terms of the Creative Commons Attribution Non-Commercial License (CC BY-NC 4.0). 


\begin{abstract}
.
BACKGROUND: This present study was designed to follow up 82 patients among 115 MDS patients registered in study ODK0801 for 5 years, to analyze the relationship between the WT1 mRNA expression level and prognosis.

OBJECTIVE: This study aimed to investigate the clinical utility of WT1 mRNA expression levels.

METHODS: After study ODK-0801, we investigated the conditions of the same patients once a year, including any clinical and laboratory findings supporting the diagnosis, and treatment among the living patients.

RESULTS: When we assessed the survival time of 82 MDS patients by WT1 mRNA expression level, there were significant differences between the $<500$ and $\geqslant 10^{4} \mathrm{copies} / \mu \mathrm{g}$ RNA groups and between the $500-10^{4}$ and $\geqslant 10^{4}$ copies $/ \mu \mathrm{g}$ RNA groups for BM levels $(p<0.01)$. Examination of the time of freedom from acute myeloid leukemia (AML) transformation indicated that a high WT1 mRNA expression level $\left(>10^{4}\right.$ copies/ $\mu$ g RNA) was a strong prognostic factor for a short time to AML transformation.
\end{abstract}

CONCLUSION: The results indicate that the tumorigenesis of MDS is likely to originate at the stem cell level, suggesting that the WT1 mRNA level measurement in the BM is an effective prognostic marker in patients with MDS.

keywords: Myelodysplastic syndromes, follow-up study, Wilms tumor 1 mRNA expression level, peripheral blood, bone marrow, IPSS, WPSS, IPSS-R

\section{Introduction}

High expression levels of Wilms tumor 1 (WT1) mRNA have been reported not only in patients with acute myeloid leukemia (AML), but also in those with myelodysplastic syndrome (MDS) [1-7]. MDS is a generic name given to a group of diseases presenting with cytopenia and a preleukemic condition of unknown cause, and some patients with MDS progress to AML. Measurement of WT1 mRNA levels in the peripheral blood (PB) of patients with MDS was shown to offer useful information for the diagnosis of disease stage and assessment of prognosis, similar to AML patients.

WT1 mRNA expression levels in the PB and bone marrow (BM) increase as the MDS disease stage progresses and are correlated with the International Prognostic Scoring System (IPSS) score and World Health Organization (WHO) classification-based Prognostic Scoring System (WPSS) score [8,9]. The increase in WT1 mRNA expression levels in the PB and BM also leads to greater risk of progression to AML. In addition, a relationship between WT1 mRNA expression levels in the PB and BM and chromosomal aberrations was also seen as they were both correlated with the proportion of myeloblasts and were high in a poor chromosomal karyotype group [10].

This present study was designed to follow up 82 patients, who gave secondary consent, among 115 MDS patients registered in study ODK-0801 at 17 medical institutions listed in Table 1. The patients were followed up for 5 years up to 2014 to analyze in detail the relationship between the WT1 mRNA expression level and prognosis.

\section{Materials and methods}

\subsection{Ethics}

This study was conducted in compliance with the ethical principles based on the Declaration of Helsinki, Pharmaceutical Affairs Law, relevant ministerial ordinances, notifications, and ethical guidelines related to clinical studies, and the protocols of this study. The approval of institutional review boards or ethics committees of each medical institution participating in this study was obtained beforehand.

Written consent was obtained from the patients after sufficient explanation from an investigator or subinvestigator, with the use of explanatory documents for the informed consent form, and after confirming that they fully understood the contents and were willing to participate in the study.

\subsection{Patients}

The 82 MDS patients studied (2008 WHO-based MDS subtypes: refractory cytopenia with unilineage dysplasia [RCUD], 29; refractory anemia with ringed sideroblasts [RARS], 1; refractory cytopenia with multilineage dysplasia [RCMD], 26; refractory anemia with excess blasts [RAEB]-1, 8; RAEB-2, 13; isolated $\operatorname{del}(5 \mathrm{q})$ chromosomal abnormality [5q-syndrome], 1; MDS, unspecified [MDS-U], 3; unclassified, 1) provided secondary consent to participate in this study and were drawn from among those who participated in study ODK-0801 conducted from December 2008 to September 2009 (Table 2). Note that the design of ODK-0801 was a one-point study, and thus the first 
Table 1

Study sites and investigators

\begin{tabular}{lll}
\hline Medical Institution & Department & Primary investigators \\
\hline Sapporo Hokuyu Hospital & Hematology & Kiyotoshi Imai \\
Dokkyo Medical University School of Medicine & Hematology and Oncology & Kinuko Mitani \\
Jichi Medical University & Hematology & Keiya Ozawa \\
Saiseikai Maebashi Hospital & Hematology & Toru Sakura \\
Tokyo Medical University Hospital & Hematology & Kazuma Ohyashiki \\
Nippon Medical School & Hematology & Kazuo Dan, Hideto Tamura \\
Nihon University School of Medicine & Hematology and Rheumatology & Jin Takeuchi, Yoshihiro Hatta \\
The university of Tokyo Hospital & Hematology and Oncology & Mineo Kurokawa \\
Tokyo Women's Medical University & Hematology & Toshiko Motoji, Junji Tanaka \\
NTT Medical Center Tokyo & Hematology & Kensuke Usuki \\
Nagoya University Graduate School of Medicine & Hematology and Oncology & Tomoki Naoe, Hitoshi Kiyoi \\
Fujita Health University School of Medicine & Hematology & Nobuhiko Emi \\
Osaka University Graduate School of Medicine & Hematology and Oncology & Hirohiko Shibayama \\
Kinki University Faculty of Medicine & Hematology and Rheumatology & Yasuyoshi Morita \\
Hyogo College of Medicine & Hematology & Hiroyasu Ogawa \\
Kurashiki Central Hospital & Haematology/Oncology & Yasunori Ueda \\
Nagasaki University Graduate School of Biomedical Sciences Atomic & Hematology & Yasushi Miyazaki \\
Bomb Disease Institute & & \\
\hline
\end{tabular}

consent was obtained for it (see Reference 10). The current study is a follow-up to ODK-0801 and includes data on the prognosis of individual patients.

\subsection{Methods}

After study ODK-0801, from September 2010 we investigated whether the same patients were living or deceased once a year, as well as the disease stage, clinical and laboratory findings supporting the diagnosis, and treatment among the living patients. For deceased patients, we investigated the date and cause of death. In addition, WT1 mRNA expression levels in the PB were measured according to the 2008 WHObased MDS subtype of individual patients at least once a year, and, if possible, they were also measured in the BM.

\subsection{Measurement of WT1 mRNA levels}

RNA was extracted from leukocytes in the PB and from nucleated cells in the BM using an RNeasy Mini Kit (Qiagen, Valencia, CA, USA), and WT1 mRNA was measured using a WT1 mRNA Assay Kit (Otsuka Pharmaceutical Co., Ltd., Tokyo, Japan) [11]. cDNA was synthesized from $1 \mu \mathrm{g}$ of extracted RNA by reverse transcription using the random hexamer method. Levels of WT1 and GAPDH mRNA were calculated using real-time PCR of the synthesized cDNA. COBAS TaqMan48 (Roche Molecular Diagnostics, Pleasanton, CA, USA) was used in real-time PCR, and levels of WT1 and GAPDH mRNA in the samples were calculated, respectively, by simultaneous re- actions of cDNA synthesized by reverse transcription and reference standards of known concentrations. The lower limit of measurement for this WT1 mRNA assay kit was 50 copies/ $\mu$ g RNA.

\subsection{Statistical analysis}

Differences between two groups of data of continuous variables were analyzed by Student's t-test. Differences between three or more groups of data on continuous variables were analyzed by one-way ANOVA with the Tukey-Kramer multiple-comparison test. Differences in categorical variables were evaluated using Fisher's exact test. Statistical analysis was performed using SAS version 9.3 (SAS Institute, Cary, NC). A Cox proportional hazard model was used to investigate survival time in MDS patients and its predictive factors and to estimate corresponding hazard ratios (HRs) and $95 \%$ confidence intervals (CIs). Both the categorical and continuous variables mentioned below were calculated based on the actual values stated in study ODK-0801.

\subsubsection{When using the WPSS}

The 6 multivariable predictive factors were the following categorical variables: gender (female: 0 , male: 1), myeloblasts (<5\%: 0, 5-10\%: 0.5, 11-20\%: 1.5), WHO subtype (refractory anemia [RA], RARS, MDS associated with 5q- syndrome: 0, RCMD, RCMD and ringed sideroblasts [RCMD-RS]: 1, RAEB-1: 2, RAEB-2: 3), transfusion requirement (no: 0, regular: 1 ), karyotype (good: 0 , intermediate: 1 , poor: 2 ), and 
WPSS score (very low: 0 , low: 1 , intermediate: 2 , high: 3-4, very high: 5-6).

The 6 multivariable predictive factors were the following continuous variables: white blood cell count (WBC), WT1 in the BM, blasts in the PB, hemoglobin $(\mathrm{Hb}), \mathrm{WT} 1$ in the PB, and platelet count. Each parameter that continues was standardized by changing to average $=0$ and dispersion $=1$ after calculating the mean and the standard deviation (SD), and applying to the formula (data - mean)/SD in regard to each parameter.

\subsubsection{When using the IPSS}

The 6 multivariable predictive factors were the following categorical variables: gender (female: 0 , male: 1), myeloblasts (< 5\%: $0,5-10 \%: 0.5,11-20 \%: 1.5$, 21-30\%: 2), French-American-British (FAB) subtype (RA: 1, RARS: 2, RAEB: 3, RAEB-t: 4), number of cytopenic lineages (0-1: 0, 2-3: 0.5), karyotype (good: 0 , intermediate: 0.5 , poor: 1 ), and IPSS score (low: 0 , intermediate [Int]-1: 0.5-1.0, intermediate-2: 1.5-2.0, high: $>2.5$ ).

The 6 multivariable predictive factors were the following continuous variables: WBC, WT1 in the BM, blasts in the PB, Hb, WT1 in the PB, and platelet count. Each parameter that continues was standardized.

\subsubsection{When using the Revised IPSS}

The 7 multivariable predictive factors were the following categorical variables: gender (female: 0 , male: $1)$, myeloblasts $(<2 \%: 0,2 \%-<5 \%: 1,5-10 \%: 2$, $>10 \%$ : 3), FAB subtype (RA: 1, RARS: 2, RAEB: 3, RAEB-t: 4), Hb (>10 g/dL: 0, 8-< 10 g/dL: 1, < 8 g/dL: 1.5), karyotype (very good: 0 , good: 1 , intermediate: 2 , poor: 3 , very poor: 4$)$, platelet count $(>$ $\left.10^{5} / \mu \mathrm{L}: 0,5-<10^{5} / \mu \mathrm{L}: 0.5,<10^{5} / \mu \mathrm{L}: 1\right)$, and Revised IPSS (IPSS-R) score (very low: $<1.5$, low: $>$ 1.5-3, intermediate: $>3-4.5$, high: $>4.5-6$, very high: $>6)$.

The 7 multivariable predictive factors were the following continuous variables: WBC, ferritin level, lactate dehydrogenase (LDH) level, WT1 in the BM, blasts in the PB, absolute neutrophil count (ANC), and WT1 in PB. Each parameter that continues was standardized.

To investigate the relationship between WT1 mRNA expression levels in the $\mathrm{PB}$ and $\mathrm{BM}$ and survival time, a survival curve was determined using the KaplanMeier method, and the log-rank $\mathrm{p}$ value in TukeyKramer's honest significant difference (HSD) test was determined to examine homogeneity among groups. The level of statistical significance was set at $p<0.05$ in all cases.
Table 2

Characteristics of patients

\begin{tabular}{|c|c|}
\hline Male/Female & $50 / 32$ \\
\hline \multicolumn{2}{|l|}{ Age $(y, \pm S D)$} \\
\hline Male & $65.7 \pm 15.4$ \\
\hline Female & $63.5 \pm 13.5$ \\
\hline \multicolumn{2}{|c|}{ Diagnosis (WHO 2008 classification) } \\
\hline RCUD & 29 \\
\hline RARS & 1 \\
\hline RCMD & 26 \\
\hline RAEB-1 & 8 \\
\hline RAEB-2 & 13 \\
\hline $5 q$-syndrome & 1 \\
\hline MDS-U & 3 \\
\hline Unclassified & 1 \\
\hline \multicolumn{2}{|c|}{ Diagnosis (FAB classification) } \\
\hline RA & 55 \\
\hline RARS & 6 \\
\hline RAEB & 20 \\
\hline RAEB-t & 1 \\
\hline \multicolumn{2}{|l|}{ WPSS } \\
\hline Very low & 19 \\
\hline Low & 23 \\
\hline Intermediate & 15 \\
\hline High & 16 \\
\hline Very high & 8 \\
\hline Unknown & 1 \\
\hline \multicolumn{2}{|l|}{ IPSS } \\
\hline Low & 16 \\
\hline Intermediate-1 & 47 \\
\hline Intermediate- 2 & 11 \\
\hline High & 8 \\
\hline \multicolumn{2}{|l|}{ IPSS-R } \\
\hline Very low & 8 \\
\hline Low & 29 \\
\hline Intermediate & 21 \\
\hline High & 11 \\
\hline Very high & 13 \\
\hline
\end{tabular}

RCUD: refractory cytopenia with unilineage dysplasia; RARS: refractory anemia with ringed sideroblasts; RCMD: refractory cytopenia with multilineage dysplasia; RAEB-1: refractory anemia with excess blasts-1; RAEB-2: refractory anemia with excess blasts-2; 5qsyndrome: myelodysplastic syndrome with isolated del(5q); MDS$\mathrm{U}$ : myelodysplastic syndrome, unclassifiable; RA: refractory anemia; RAEB: refractory anemia with excess blasts; RAEB-t: refractory anemia with excess blasts in transformation.

\section{Results}

\subsection{Information about clinical and cytogenetic data}

Information about clinical and cytogenetic data were obtained from 82 MDS patients (Table 3).

As shown in Table 3, in low-grade MDS, multilineage dysplasia and abnormal cytogenetics were not completely associated with clinical data (White blood cell count (WBC), Hemoglobin, Platelet count and Absolute neutrophil count (ANC)). However, risk group (IPSS, WPSS, and IPSS-R) were associated with clinical data. Significant difference $(p<0.05)$ was 


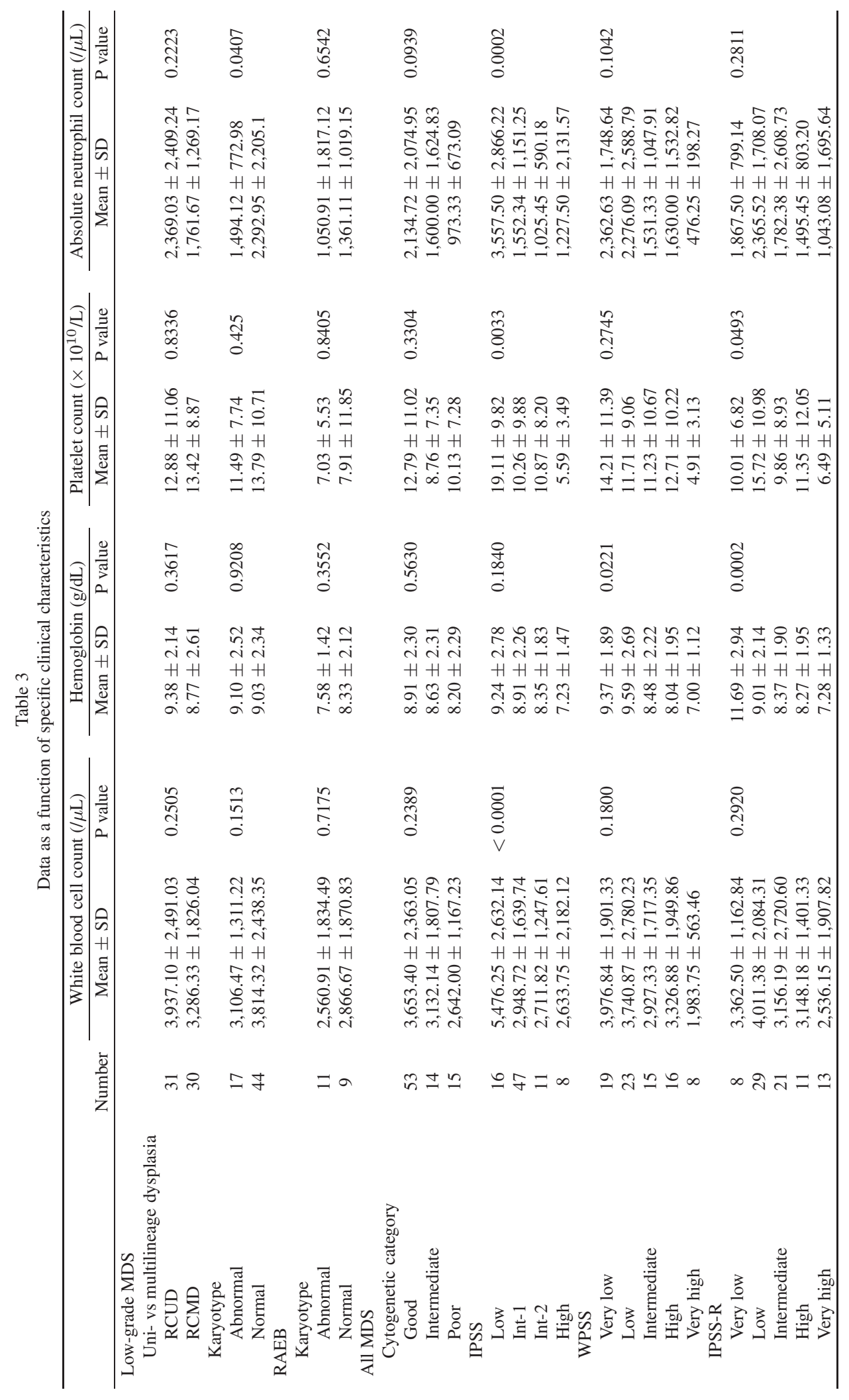


Table 4

Analysis of Cox proportional hazard models a) WHO classification: WPSS

\begin{tabular}{lccc}
\hline Parameter & Hazard ratio & $95 \%$ CI & p value \\
\hline WPSS score & 1.798 & $1.428-2.264$ & $<0.0001^{*}$ \\
WBC & 2.067 & $1.456-2.936$ & $<0.0001^{*}$ \\
WT1 in BM & 1.667 & $1.220-2.277$ & $0.0013^{*}$ \\
Blasts in PB & 1.307 & $1.074-1.590$ & $0.0075^{*}$ \\
Gender & 2.883 & $1.250-6.648$ & $0.0130^{*}$ \\
\hline \multicolumn{5}{c}{} \\
b) FAB classification: IPSS & & \\
\hline Parameter & Hazard ratio & $95 \%$ CI & p value \\
\hline IPSS score & 2.430 & $1.601-3.687$ & $<0.0001^{*}$ \\
WBC & 2.079 & $1.469-2.944$ & $<0.0001^{*}$ \\
Blasts in PB & 1.406 & $1.145-1.725$ & $0.0011^{*}$ \\
WT1 in BM & 1.585 & $1.155-2.175$ & $0.0043^{*}$ \\
Gender & 3.065 & $1.303-7.211$ & $0.0103^{*}$ \\
Hemoglobin & 0.618 & $0.394-0.972$ & $0.0371^{*}$ \\
\hline \multicolumn{5}{c}{} & \\
c) FAB classification: IPSS-R & & p value \\
\hline Parameter & Hazard ratio & $95 \%$ CI \\
\hline IPSS-R score & 1.547 & $1.297-1.845$ & $<0.0001^{*}$ \\
WBC & 1.990 & $1.431-2.767$ & $<0.0001^{*}$ \\
Blasts in PB & 1.374 & $1.133-1.665$ & $0.0012^{*}$ \\
WT1 in BM & 1.550 & $1.139-2.110$ & $0.0053^{*}$ \\
Gender & 2.809 & $1.211-6.518$ & $0.0162^{*}$ \\
\hline
\end{tabular}

* Selected using the stepwise method. Each parameter that continues (hemoglobin, WBC, blasts in PB, and WT1 in BM) was standardized. WPSS: World Health Organization classification-based Prognostic Scoring System; WBC: white blood cell count; BM: bone marrow; PB: peripheral blood; FAB: French-American-British; IPSS: International Prognostic Scoring System; IPSS-R: Revised International Prognostic Scoring System.

observed only in Karyotype of ANC in low-risk MDS (RCUD and RCMD) of the WHO classification. On the other hand, significant differences were not observed in any items (WBC, Hemoglobin, Platelet count and ANC) in the RAEB group, a high-risk group.

Furthermore, in all the MDS patients, significant differences were observed $(p<0.05)$ in 3 items, WBC, Platelet count and ANC, in IPSS risk group, and significant difference was observed only in Hemoglobin in the WPSS risk group. In the IPSS-R risk group, a significant differences were observed in Hemoglobin and Platelet count.

\subsection{Prognostic factors for survival time}

Predictive factors of survival time were investigated based on the data at the time of registration in study ODK-0801. As a result, the WBC count in the PB (HR $=2.067, \mathrm{CI}=1.456-2.936, p<0.0001)$, WPSS score $(\mathrm{HR}=1.798, \mathrm{CI}=1.428-2.264, p<0.0001), \mathrm{WT} 1$ mRNA expression levels in the BM $(\mathrm{HR}=1.667, \mathrm{CI}$ $=1.220-2.277, p=0.0013)$, blasts in the $\mathrm{PB}(\mathrm{HR}=$ $1.307, \mathrm{CI}=1.074-1.590, p=0.0075)$, and gender
$(\mathrm{HR}=2.883, \mathrm{CI}=1.250-6.648, p=0.0130)$ were selected from the WPSS from the categories including gender, age, WT1 mRNA expression level in the PB, WT1 mRNA expression level in the BM, WBC count in the $\mathrm{PB}, \mathrm{Hb}$ level, platelet count, blast count in the $\mathrm{PB}$, myeloblasts, WHO subtype, blood transfusion dependency, chromosomal karyotype, and WPSS score (Table 4a).

In a similar analysis using the FAB classification, the IPSS score $(\mathrm{HR}=2.430, \mathrm{CI}=1.601-3.687, p<$ $0.0001)$, WBC count in the PB $(\mathrm{HR}=2.079, \mathrm{CI}=$ 1.469-2.944, $p<0.0001$ ), blasts in the PB (HR = $1.406, \mathrm{CI}=1.145-1.725, p=0.0011)$, WT1 mRNA expression levels in the $\mathrm{BM}(\mathrm{HR}=1.585, \mathrm{CI}=1.155$ $2.175, p=0.0043)$, gender $(\mathrm{HR}=3.065, \mathrm{CI}=1.303$ 7.211, $p=0.0103)$, and Hb level $(\mathrm{HR}=0.618, \mathrm{CI}=$ $0.394-0.972, p=0.0371$ ) were selected (Table 4b). When the IPSS-R was used instead of the IPSS in the analysis based on the FAB classification, the same prognostic factors were selected, except that the IPSS score was changed to the IPSS-R score [12] (Table 4c). The range of each HR by which variable selection was made was 1.13 to 6.52 . WT1 mRNA expression level in the BM was selected as a significant prognostic factor for survival time in both classifications.

\subsection{Estimation based on the analysis of Cox proportional hazard models by IPSS-R risk category}

Survival times were estimated by the five selected parameters in which WT1 mRNA expression levels varied (500, 50,000, 100,000, and 150,000 copies), as shown in Fig. 1, whereas four other parameters in the analysis using the IPSS-R risk categories (IPSS-R score, WBC count in the PB, myeloblasts in the PB, and gender) were constant. The median survival period of patients with WT1 mRNA expression levels of $500,50,000,100,000$, and 150,000 copies was more than $60,45,17.5$, and 12 months, respectively, demonstrating a good correlation with prognosis. The survival time became shorter as the WT1 mRNA expression level in the BM was multiplied by higher factors (Fig. 1).

\subsection{Relationship between WT1 mRNA expression levels and overall survival rate}

The 82 patients were classified into 3 groups based on their WT1 mRNA expression levels in the PB and $\mathrm{BM}$; a survival curve (censored at the point of allo- 


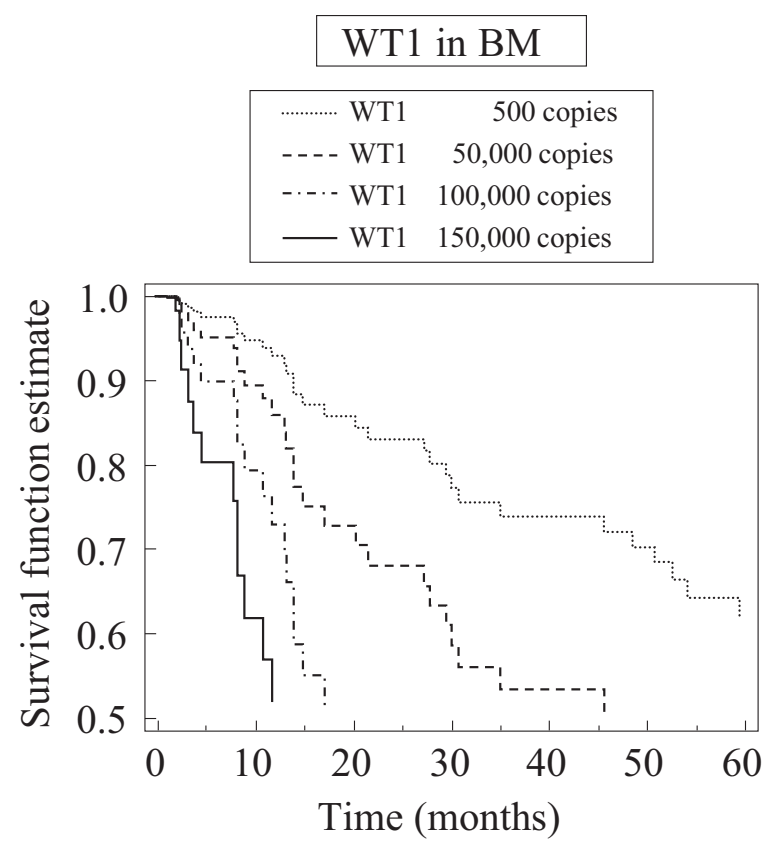

Fig. 1. Estimation of survival time based on the analysis of Cox proportional hazard models by IPSS-R risk category.

geneic transplantation) was prepared using the KaplanMeier method, and the groups were compared for homogeneity in the log-rank test. WT1 mRNA expression levels in the PB were classified into the following 3 groups: $<50$ copies $/ \mu$ g RNA; $50-10^{4}$ copies $/ \mu \mathrm{g}$ RNA; and $\geqslant 10^{4}$ copies/ $\mu$ g RNA. WT1 mRNA expression levels in the BM were classified into the following 3 groups: $<500$ copies; $500-10^{4}$ copies; and $\geqslant 10^{4}$ copies.

When we assessed the survival time of 82 MDS patients by WT1 mRNA expression level, significant differences were detected between the $<500$ group and $\geqslant$ $10^{4}$ group and between the 500-10 $0^{4}$ group and $\geqslant 10^{4}$ group for BM levels $(p<0.01)$. For WT1 mRNA expression levels in the $\mathrm{PB}$, significant differences were also detected between the $<50$ group and $\geqslant 10^{4}$ group $(p<0.01)$. The overall survival rate differed significantly among the groups classified by WT1 mRNA expression levels in both the PB and BM, demonstrating a shortening of survival time with increasing WT1 mRNA expression levels (Fig. 2a).

The survival time of 55 RA patients in the FAB classification was not significantly different when they were grouped according to their PB and BM WT1 mRNA expression levels (data not shown). The same analysis of 47 patients in the IPSS Int-1 group was assessed according to their WT1 mRNA expression levels, and a significant difference was detected between the $<500$ group and $\geqslant 10^{4}$ group and between the $500-10^{4}$ group and $\geqslant 10^{4}$ group for BM levels $(p<$ 0.05 ). This finding indicates a shortening of survival time with increasing WT1 mRNA expression levels in the BM (Fig. 2b). Conversely, the survival time of 56 patients in the lower-risk WPSS groups (very low, low, and intermediate) was not significantly different when they were grouped according to their PB and BM WT1 mRNA expression levels (data not shown). Further, when the survival period of 58 patients in the lowrisk IPSS-R groups (very low, low, and intermediate) was assessed according to their WT1 mRNA expression levels, no significant difference was detected in either the PB or BM (data not shown).

As shown in Fig. 3a, the conventionally defined 5year probability of Leukemia-free survival (LFS) in 82 MDS patients was $77 \%$ in both PB and BM.

When we assessed the time of freedom from AML transformation in 82 MDS patients by WT1 mRNA expression level, significant differences were observed between the $<500$ group and $\geqslant 10^{4}$ group and between the $500-10^{4}$ group and $\geqslant 10^{4}$ group for BM levels $(p<$ $0.01)$. In the case of WT1 mRNA expression levels in the PB, significant differences were also observed between the $<50$ group and $\geqslant 10^{4}$ group and between the $50-10^{4}$ group and $\geqslant 10^{4}$ group $(p<0.01)$. Examination of the time of freedom from AML transformation indicated that a high WT1 mRNA expression level (> $10^{4}$ copies/ $\mu \mathrm{g}$ RNA) was a strong prognostic factor for a short time to AML transformation (Fig. 3b).

\subsection{Relationship between WT1 mRNA expression levels and IPSS-R risk group}

WT1 mRNA expression in PB and BM for each IPSS-R risk group were compared in the 82 patients, who gave secondary consent, among 115 MDS patients registered in study ODK-0801. A tendency for WT1 mRNA expression to increase in both PB and BM was observed in each IPSS-R risk group as the risk of transformation to AML increased from very low to very high. Significant differences $(p<0.05)$ in WT1 mRNA expression were observed in risk groups between very low and high, very low and very high, low and high, low and very high, and intermediate and very high in PB samples; and between very low and high, very low and very high, low and high, and low and very high in BM samples (Fig. 4).

When lines indicating WT1 mRNA expression levels of 50 and $10^{4}$ copies/ $\mu$ g RNA were drawn on scatter diagrams for the IPSS-R risk groups in this study, 

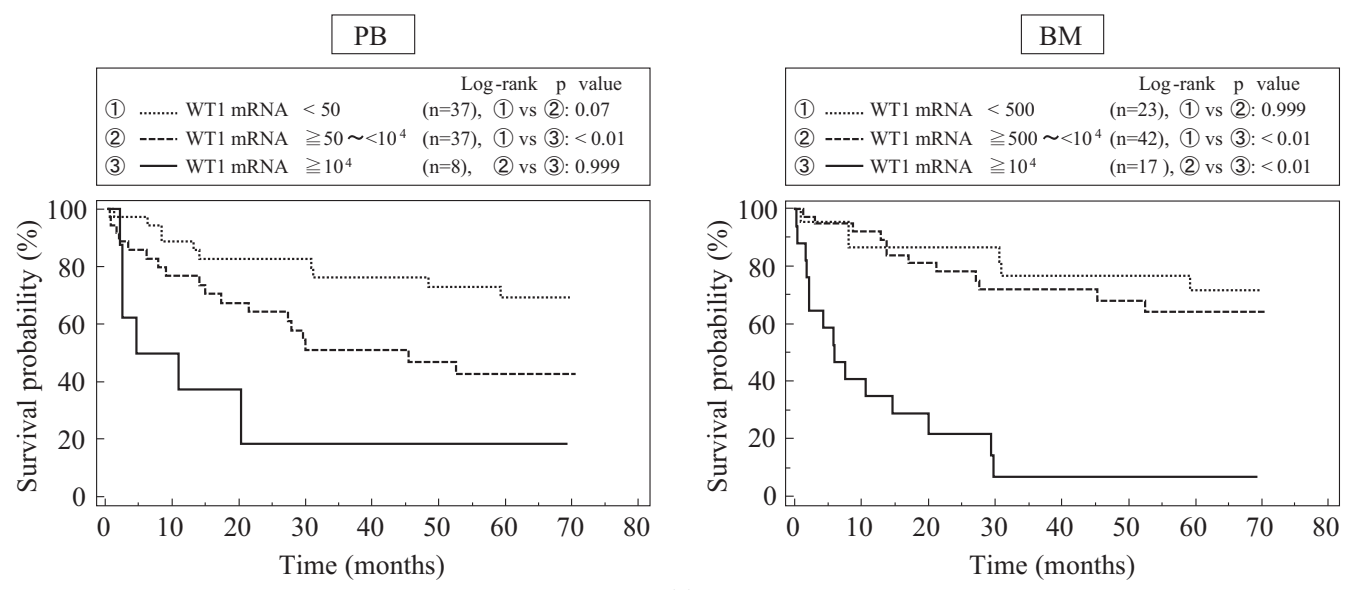

(a)
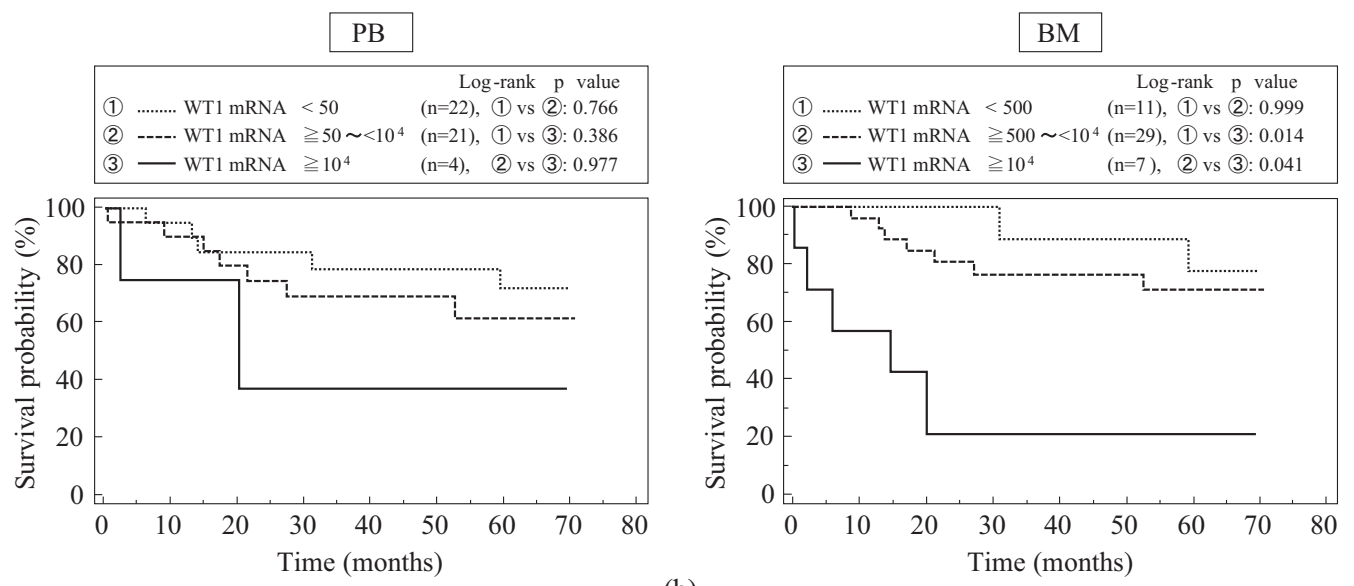

(b)

Fig. 2. WT1 mRNA expression levels in peripheral blood (PB) and bone marrow (BM) and overall survival of MDS patients. a: All MDS patients $(n=82)$, b: IPSS Int-1 patients $(n=47)$.

WT1 mRNA expression levels in the low and Intermediate groups were distributed widely from a low level of $<50$ copies $/ \mu \mathrm{g}$ RNA to a high level of $\geqslant 10^{4}$ copies/ $\mu \mathrm{g}$ RNA. In this study, the overall survival rate decreased significantly in the group with WT1 mRNA expression levels of $\geqslant 10^{4}$ copies/ $\mu \mathrm{g}$ RNA in the BM compared with the survival rate in the other two groups of Int-1 patients (Fig. 2b).

\section{Discussion}

Tamura et al. [7] classified 69 MDS patients into 3 groups according to their WT1 mRNA expression levels in the PB in a retrospective analysis and compared the survival time and disease-free survival time. They reported that both survival time and disease-free survival time were shorter in the group with high WT1 mRNA expression levels compared with the groups with lower expression levels and that WT1 mRNA expression levels in the PB were an independent prognostic factor in MDS [7]. Our prospective study results support those findings.

Hematopoietic stem cell transplantation is indicated for patients with MDS in the IPSS Int-2 and high groups, provided that they meet the conditions for transplantation. If the conditions are not met, treatment with 5-azacytidine, decitabine, or high-potency chemotherapy is attempted. While immunosuppressive therapy is indicated for patients in the IPSS low and Int-1 groups, hematopoietic stem cell transplantation is indicated if frequent blood transfusions are required.

In MDS, the timing of transplantation has always been an issue. Cutler et al. [13] investigated the optimal timing for BM transplantation using myeloablative conditioning of human leukocyte antigen (HLA)identical sibling donors in MDS patients and reported that a significant improvement in the survival rate 

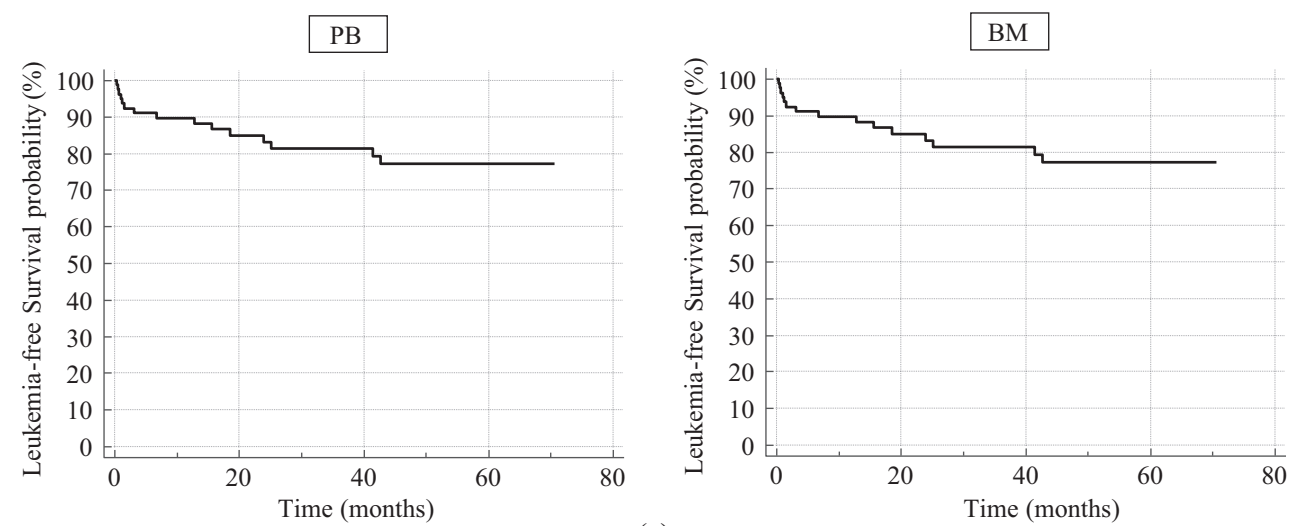

(a)
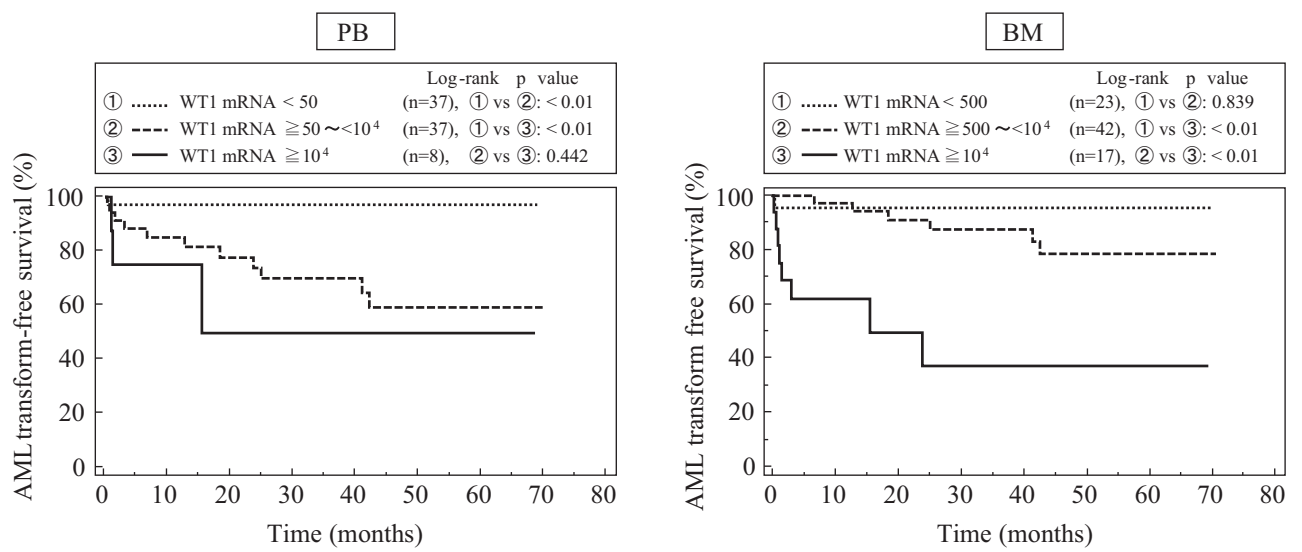

(b)

Fig. 3. Leukemia-free survival and WT1 mRNA expression levels in PB and BM and time of freedom from AML transformation in 82 MDS patients. a: Leukemia-free survival $(n=82)$, b: Time of freedom from AML transformation $(n=82)$.

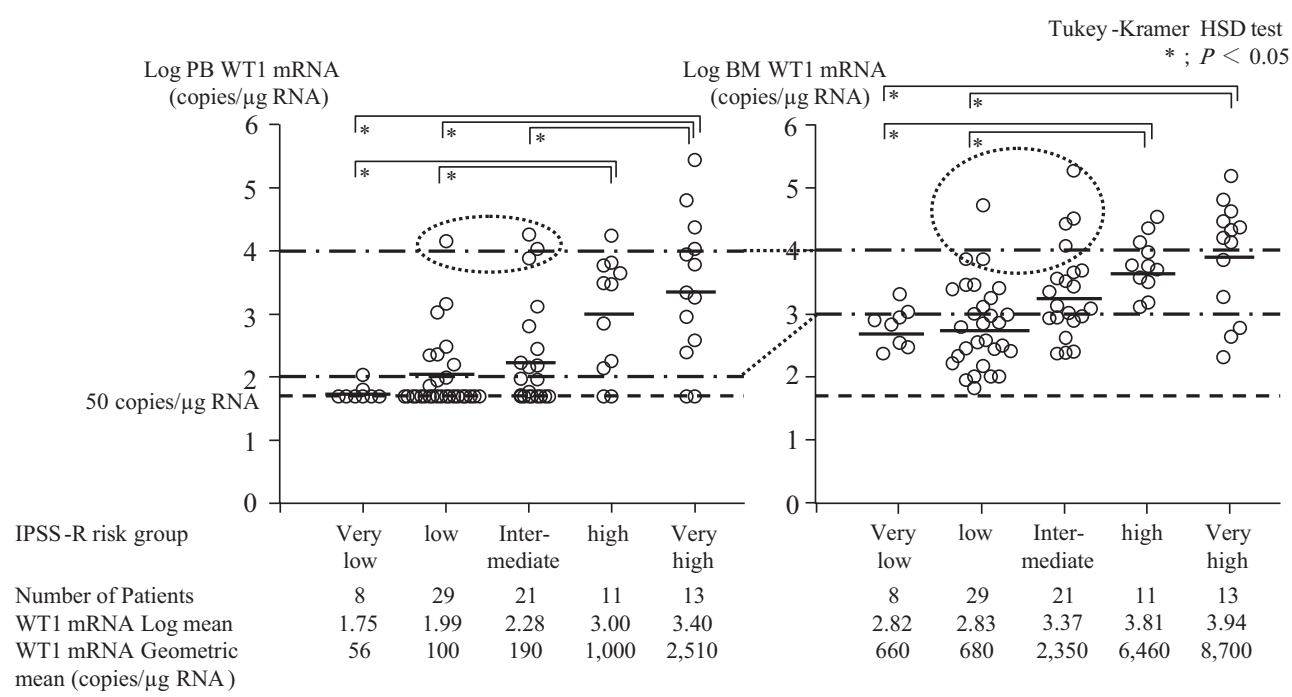

Fig. 4. WT1 mRNA expression levels in PB and BM by IPSS-R risk group in 82 MDS patients. 
could be expected following transplantation in the early stage after diagnosis in patients in the Int-2 and high-risk groups, although in the low- and Int-1-risk groups, the highest survival rate was expected by performing transplantation when disease progression was noted during careful monitoring of disease course after diagnosis rather than immediately after diagnosis. However, those results were restricted to HLAidentical sibling donor transplantation, and the quality of patients' lives after transplantation was not taken into consideration.

De Witte et al. [14], on the other hand, investigated the results of transplantation in 374 MDS patients with RA or RARS using multivariate analysis and showed that, while the degree of pretreatment or donor type had no effect on the results of transplantation, transplantation in recent years, younger patient age, and a short period from diagnosis to transplantation may result in a better outcome. However, deterioration of the quality of life after transplantation and complications in the chronic stage were not mentioned in that report, and it is still difficult to make a decision on the indication for transplantation in the low and Int-1 groups.

WT1 mRNA expression levels in the Int-1 group were distributed widely from a low level of $<50$ copies/ $\mu \mathrm{g}$ RNA to a high level of $\geqslant 10^{4}$ copies $/ \mu \mathrm{g}$ RNA, as shown in Fig. 4. In this study, the overall survival rate decreased significantly in the group with WT1 mRNA expression levels of $\geqslant 10^{4}$ copies $/ \mu \mathrm{g}$ RNA in the BM compared with the survival rate in the other two groups of Int-1 patients (Fig. 2b). Patients with a WT1 mRNA expression level of $10^{4}$ copies $/ \mu \mathrm{g}$ RNA in the BM had a poor prognosis, which also supports the hypothesis that patients with high WT1 mRNA expression levels will have a poor prognosis (Fig. 1).

Ommen et al. reported that the WT1 mRNA level in the $\mathrm{BM}$ is superior to that in the $\mathrm{PB}$ in predicting prognosis, as observed in AML patients from whom samples were available within 3 months prior to clinical relapse. They proposed the following monitoring method to identify differing relapse kinetics patterns: in the BM, almost $100 \%$ of clinical relapses can be predicted based on WT1 mRNA level measured in the period 3 to 4 months before relapse; and in the $\mathrm{PB}, 80 \%$ of the cases can be predicted based on the WT1 mRNA level measured bimonthly [15].

The WT1 mRNA level in the PB is therefore an effective prognostic marker in patients with AML. The tumorigenesis of AML is thought to be at the stem cell level, suggesting that the measurement of the WT1
mRNA expression level in the BM is not necessary. However, the analysis in this study revealed that the WT1 mRNA expression level in the BM reflects trends in the effectiveness of treatment and the number of residual tumor cells. This finding indicates that the tumorigenesis of MDS is also likely to originate at the stem cell level, and thus the measurement of the WT1 mRNA level in the BM is an effective prognostic marker in patients with MDS.

In the treatment of IPSS Int-1 patients, transplantation is not the first-line treatment according to the guidelines of the Japan Society for Hematopoietic Cell Transplantation [16], although it may be considered for patients who meet the transplantation conditions. However, the results of this study showed a poor prognosis in patients with high WT1 mRNA expression levels, particularly in those with $>10^{4}$ copies $/ \mu \mathrm{g}$ RNA, among patients in the Int-1 group, who are thought to be at lower risk, suggesting that transplantation should be performed in this group, similar to Int-2 patients.

In the IPSS-R very low and low risk subgroups encompassed those who had been classified as IPSS low and Int-1. The IPSS-R intermediate category was composed of about 75\% IPSS Int-1 [12]. So, in the treatment of IPSS-R Low and IPSS-R Intermediate patients with WT1 mRNA expression level of $\geqslant 10^{4}$ copies $/ \mu \mathrm{g}$ RNA may be considered as patients who are indicated to be in the transplantation setting.

On the basis of the above results, it is concluded that mRNA expression levels of WT1 in the PB and BM of MDS patients are useful prognostic indicators and can contribute to determining the appropriate therapeutic strategy, including allogeneic hematopoietic stem cell transplantation, which is the only radical cure for MDS. Furthermore, the measurement of WT1 mRNA expression levels is expected to contribute to the improvement of prognosis, such as prolongation of survival time, in MDS patients.

\section{Acknowledgments}

This study was funded by Otsuka Pharmaceutical Co., Ltd., Tokyo, Japan. We thank all participating institutions and physicians, not only the authors, but also the following investigators, for their support for the study.

The participating institutions were: Chisato Mizutani (Department of Haematology/Oncology, Transfusion and Haemapheresis Center, Kurashiki Central Hospital, Okayama); Department of Hematol- 
ogy and Oncology, University of Tokyo Graduate School of Medicine, Tokyo; Department of Hematology and Rheumatology, Nihon University School of Medicine, Tokyo; Division of Hematology, Department of Medicine, Nippon Medical School, Tokyo; Department of Hematology and Oncology, Osaka University Graduate School of Medicine, Osaka; Rika Kihara and Yuichi Ishikawa (Department of Hematology and Oncology, Nagoya University Graduate School of Medicine, Nagoya); Ko Sasaki (Department of Hematology and Oncology, Dokkyo Medical University School of Medicine, Tochigi); Department of Hematology, Fujita Health University School of Medicine, Aichi; Department of Hematology, Tokyo Women's Medical University, Tokyo; Michiko Kida (Division of Hematology, NTT Medical Center Tokyo, Tokyo); Satoru Takada (Department of Hematology, Saiseikai Maebashi Hospital, Gunma); Department of Hematology, Tokyo Medical University Hospital, Tokyo; Division of Hematology, Department of Internal Medicine, Hyogo College of Medicine, Hyogo; Division of Hematology, Department of Medicine, Jichi Medical University, Tochigi; Department of Hematology, Sapporo Hokuyu Hospital, Sapporo; Department of Hematology, Atomic Bomb Disease Institute, Nagasaki University Graduate School of Biomedical Sciences, Nagasaki; and Division of Hematology and Rheumatology, Department of Internal Medicine, Kinki University Faculty of Medicine, Osaka-Sayama.

\section{Conflict of interest}

S.K., Y.U., Y.N., H. Shibayama, K.O., K.S., Y.A., K.U., Y.I., M.O., T.S., T.H., A.M. and K.T. received research funding from Otsuka Pharmaceutical Co., Ltd. K.M., T.N. and H. Sugiyama received personal fees from Otsuka Pharmaceutical Co., Ltd., during the conduct of the study. K.K. and D.K. are employees of Otsuka Pharmaceutical Co., Ltd. F.T. declares no conflict of interest.

\section{References}

[1] H. Tamaki, H. Ogawa, K. Ohyashiki, J.H. Ohyashiki, H. Iwama, K. Inoue, T. Soma, Y. Oka, T. Tatekawa, Y. Oji, A. Tsuboi, E.H. Kim, M. Kawakami, K. Fuchigami, M. Tominaga, K. Toyama, K. Aozasa, T. Kishimoto and H. Sugiyama, The Wilms' tumor gene WT1 is a good marker for diagnosis of disease progression of myelodysplastic syndromes, Leukemia 13 (1999), 393-9.
[2] D. Cilloni, E. Gottardi, F. Messa, M. Fava, P. Scaravaglio, M. Bertini, M. Girotto, C. Marinone, D. Ferrero, A. Gallamini, A. Levis and G. Saglio, Significant correlation between the degree of WT1 expression and the International Prognostic Scoring System score in patients with myelodysplastic syndromes, J Clin Oncol 21 (2003), 1988-95.

[3] P. Patmasiriwat, G. Fraizer, H. Kantarjian and G.F. Saunders, WT1 and GATA1 expression in myelodysplastic syndrome and acute leukemia, Leukemia 13 (1999), 891-900.

[4] P. Bader, C. Niemeyer, G. Weber, T. Coliva, V. Rossi, H. Kreyenberg, A. Gerecke and A. Biondi, WT1 gene expression: Useful marker for minimal residual diseases in childhood myelodysplastic syndromes and juvenile myelomonocytic leukemia, Eur J Haematol 73 (2004), 25-8.

[5] K. Tamura, T. Kanazuwa, M. Suzuki, M. Koitabashi, C. Ogawa and A. Morikawa, Successful rapid discontinuation of immunosuppressive therapy at molecular relapse after allogeneic bone marrow trasplantation in pediatric patients with myelodysplastic syndrome, Am J Hematol 81 (2006), 139-41.

[6] T. Iwasaki, C. Sugisaki, K. Nagata, K. Takagi, A. Takagi, T. Kojima, M. Ito, S. Nakamura, T. Naoe and T. Murate, Wilms' tumor 1 message and protein expression in bone marrow failure syndrome and acute leukemia, Pathol Int 57 (2007), 64551.

[7] H. Tamura, K. Dan, N. Yokose, R. Iwakiri, M. Ohta, H. Sakamaki, K. Tohyama, A. Kondo, H. Hyodo, K. Nakamura, T. Yamashita, O.A. Elisseeva, Y. Oka, Y. Oji, H. Sugiyama and K. Ogata, Prognostic significance of WT1 mRNA and anti-WT1 antibody levels in peripheral blood in patients with myelodysplastic syndromes, Leuk Res 34 (2010), 986-90.

[8] P. Greenberg, C. Cox, M.M. LeBeau, P. Fenaux, P. Morel, G. Sanz, M. Sanz, T. Vallespi, T. Hamblin, D. Oscier, K. Ohyashiki, K. Toyama, C. Aul, G. Mufti and J. Bennett, International scoring system for evaluating prognosis in myelodysplastic syndromes, Blood 89 (1997), 2079-88.

[9] L. Malcovati, U. Germing, A. Kuendgen, M.G. Della Porta, C. Pascutto, R. Invernizzi, A. Giagounidis, B. Hildebrandt, P. Bernasconi, S. Knipp, C. Strupp, M. Lazzarino, C. Aul, and M. Cazzola, Time-dependent prognostic scoring system for predicting survival and leukemic evolution in myelodysplastic syndromes, J Clin Oncol 25 (2007), 3503-10.

[10] Y. Ueda, C. Mizutani, Y. Nannya, M. Kurokawa, S. Kobayashi, J. Takeuchi, H. Tamura, K. Ogata, K. Dan, H. Shibayama, Y. Kanakura, K. Niimi, K. Sasaki, M. Watanabe, N. Emi, M. Teramura, T. Motoji, M. Kido, K. Usuki, S. Takada, T. Sakura, Y. Ito, K. Ohyashiki, H. Ogawa, T. Suzuki, K. Ozawa, K. Imai, M. Kasai, T. Hata, Y. Miyazaki, Y. Morita, A. Kanamaru, A. Matsuda, K. Tohyama, D. Koga, H. Tamaki, K. Mitani, T. Naoe, H. Sugiyama and F. Takaku, Clinical evaluation of WT1 mRNA expression levels in peripheral blood and bone marrow in patients with myelodysplastic syndromes, Leuk Lymphoma 54 (2013), 1450-8.

[11] S. Miyawaki, N. Hatsumi, T. Tamaki, T. Naoe, K. Ozawa, K. Kitamura, T. Karasuno, K. Mitani, Y. Kodera, T. Yamagami and D. Koga, Prognostic potential of detection of WT1 mRNA level in peripheral blood in adult acute myeloid leukemia, Leuk Lymphoma 51 (2010), 1855-61.

[12] P.L. Greenberg, H. Tuechler, J. Schanz, G. Sanz, G. GarciaManero, F. Sole, J.M. Bennett, D. Bowen, P. Fenaux, F. Dreyfus, H. Kantarjian, A. Kuendgen, A. Levis, L. Malcovati, M. Cazzola, J. Cermak, C. Fonatsch, M.M. Le Beau, M.L. Slovak, O. Krieger, M. Luebbert, J. Maciejewski, Silvia M.M. Magalhaes, Y. Miyazaki, M. Pfeilstocker, M. Sekeres, W.R. Sperr, R. Stauder, S. Tauro, P. Valent, T. Vallespi, A.A. Van de 
Loosdrecht, U. Germing and D. Haase, Revised International Prognostic Scoring System for myelodysplastic syndromes, Blood 120 (2012), 2454-65.

[13] C.S. Cutler, S.J. Lee, P. Greenberg, H.J. Deeg, W.S. Perez, C. Anasetti, B.J. Bolwell, M.S. Cairo, R.P. Gale, J.P. Klein, H.M. Lazarus, J.L. Liesveld, P.L. McCarthy, G.A. Milone, J.D. Rizzo, K.R. Schultz, M.E. Trigg, A. Keating, D. Weisdorf, J.H. Antin and M.M. Horowitz, A decision analysis of allogeneic bone marrow transplantation for the myelodysplastic syndromes: Delayed transplantation for low-risk myelodysplasia is associated with improved outcome, Blood 104 (2004), 579-85.

[14] T. de Witte, R. Brand, A. van Biezen, G. Mufti, T. Ruutu, J. Finke, P. von dem Borne, A. Vitek, M. Delforge, P. Alessandrino, N. Harlahakis, N. Russell, R. Martino, L. Verdonck, N.
Kroger and D. Niederwieser, Allogeneic stem cell transplantation for patients with refractory anaemia with matched related and unrelated donors: Delay of the transplant is associated with inferior survival, Br J Haematol 146 (2009), 62736.

[15] H.B. Ommen, C.G. Nyvold, K. Braendstrup, B.L. Andersen, I.B. Ommen, H. Hasle, P. Hokland and M. Ostergaard, Relapse prediction in acute myeloid leukemia patients in complete remission using WT1 as a molecular marker: Development of a mathematical model to predict time from molecular to clinical relapse and define optimal sampling intervals, $\mathrm{Br} J$ Haematol 141 (2008), 782-91.

[16] JSHCT, The Japan Society for Hematopoietic Cell Transplantation monograph, 19 (2009), 8. 